\title{
Fabrication of sharp silicon hollow microneedles by deep-reactive ion etching towards minimally invasive diagnostics
}

\author{
Yan Li ${ }^{1,2}$, Hang Zhang ${ }^{1}$, Ruifeng Yang ${ }^{1}$, Yohan Laffitte ${ }^{2}$, Ulises Schmill², Wenhan Hu', Moufeed Kaddoura²,
}

Eric J. M. Blondeel ${ }^{2}$ and Bo Cui ${ }^{1}$

\begin{abstract}
Microneedle technologies have the potential for expanding the capabilities of wearable health monitoring from physiology to biochemistry. This paper presents the fabrication of silicon hollow microneedles by a deep-reactive ion etching (DRIE) process, with the aim of exploring the feasibility of microneedle-based in-vivo monitoring of biomarkers in skin fluid. Such devices shall have the ability to allow the sensing elements to be integrated either within the needle borehole or on the backside of the device, relying on capillary filling of the borehole with dermal interstitial fluid (ISF) for transporting clinically relevant biomarkers to the sensor sites. The modified DRIE process was utilized for the anisotropic etching of circular holes with diameters as small as $30 \mu \mathrm{m}$ to a depth of $>300 \mu \mathrm{m}$ by enhancing ion bombardment to efficiently remove the fluorocarbon passivation polymer. Afterward, isotropic wet and/or dry etching was utilized to sharpen the needle due to faster etching at the pillar top, achieving tip radii as small as $5 \mu$ m. Such sharp microneedles have been demonstrated to be sufficiently robust to penetrate porcine skin without needing any aids such as an impact-insertion applicator, with the needles remaining mechanically intact after repetitive penetrations. The capillary filling of DRIE-etched through-wafer holes with water has also been demonstrated, showing the feasibility of use to transport the analyte to the target sites.
\end{abstract}

\section{Introduction}

Wearable healthcare monitoring technologies have the potential for dramatically expanding the capability of data acquisition regarding an individual's health ${ }^{1,2}$. Recently, microneedle technologies have become increasingly interesting for unleashing the potential to minimally invasively interfere with an individual's biochemistry, such as for drug delivery ${ }^{3,4}$, interstitial fluid sampling ${ }^{5,6}$, and diagnostics ${ }^{7,8}$. Microneedles require only a small area of skin to be penetrated at a limited depth, resulting in

Correspondence: Eric J. M. Blondeel (eric@exvivo.com) or

Bo Cui (bcui@uwaterloo.ca)

1 Department of Electrical and Computer Engineering, University of Waterloo,

200 University Avenue West, Waterloo, ON N2L 3G1, Canada

2ExVivo Labs Inc., 3 Regina Street North, Waterloo, ON N2J 2Z7, Canada

These authors contributed equally: Yan Li, Hang Zhang minimal irritation of the dermal layers associated with pain and tissue damage.

Silicon microneedles are desirable due to their excellent biocompatibility and, in particular, mechanical properties superior to those of polymer and metal, such as a nonductile nature, high Young's Modulus, and indentation hardness enabling skin penetration without breakage in the skin 9 . Despite some concerns, silicon material revealed biocompatibility in a baseline battery of ISO 10993 physicochemical and biocompatibility tests ${ }^{10}$. With silicon implantation, comprehensive studies of the immunohistochemistry of brain tissues demonstrated that silicon devices and the byproducts of their dissolution in the intracranial space are biocompatible ${ }^{11,12}$. In particular, the Food and Drug Administration (FDA) has granted clearance for silicon devices, such as silicon microneedles (NanoPass Technologies Ltd., https://www.nanopass.

\section{(c) The Author(s) 2019}

(c) (i) Open Access This article is licensed under a Creative Commons Attribution 4.0 International License, which permits use, sharing, adaptation, distribution and reproduction cc) in any medium or format, as long as you give appropriate credit to the original author(s) and the source, provide a link to the Creative Commons license, and indicate if changes were made. The images or other third party material in this article are included in the article's Creative Commons license, unless indicated otherwise in a credit line to the material. If material is not included in the article's Creative Commons license and your intended use is not permitted by statutory regulation or exceeds the permitted use, you will need to obtain permission directly from the copyright holder. To view a copy of this license, visit http://creativecommons.org/licenses/by/4.0/. 
$\mathrm{com} /)^{13}$ and silicon Utah array electrodes (Blackrock Microsystems LLC, https://blackrockmicro.com/ electrode-main $/)^{14}$. In comparison with other microneedle materials, e.g., polymers ${ }^{15}$ and metals ${ }^{16}$, silicon has the advantages of being compatible with well-established micro/nanofabrication technologies for enabling added functionalities, such as through the monolithic integration of microneedles and complementary metal-oxidesemiconductor (CMOS) circuitry for continuous and real-time diagnostics ${ }^{17-20}$.

Interstitial fluid (ISF) holds great promise as an alternative source of biomarkers for blood plasma ${ }^{21,22}$. ISF, formed by blood transcapillary filtration, has a composition comparable to that of plasma, indicating significant untapped potential for a wide range of diagnostics. Proteomic and metabolomics analysis indicates that ISF is highly similar to both plasma and serum ${ }^{21,22}$. It has also been shown that certain biomarkers (e.g., glucose) in ISF at equilibrium have concentration levels that correlate well with those in clinically relevant blood plasma ${ }^{23,24}$. By withdrawing ISF, biomarkers of clinical interest are measured either off-line by standard commercial methods $^{22}$ or in-line by integrated biosensors ${ }^{7}$. In the latter configuration, ISF is transported through the needle lumen to the biosensor integrated on the backside of the device $^{7,23}$. Both methods necessitate a large amount of ISF volume to improve the diagnostic consistency. The epidermis largely comprises keratinocytes cells, and ISF-filled compartments are sparsely distributed in the upper region of the dermis (papillary), enveloped by the structural molecules of the interstitium matrix such as collagen frameworks ${ }^{24-26}$. The sampling of ISF using microneedles involves penetration through a deformable, elastic skin barrier, which is a challenge often resulting in incomplete needle penetration ${ }^{27,28}$. The inhomogeneity of ISF population results in inconsistent recovery and limited volume (typically submicroliter scale), and enhanced recovery necessitates fluid extraction mechanisms such as vacuum suction $^{5}$. In addition, millimeter-long needles have enabled the recovery of ISF at up to tens of microliters from the deeper dermal region (i.e., the reticular der$\mathrm{mis})^{22}$. However, ISF interrogation in the reticular dermis likely results in inevitable contact between the needle and sensory nerve endings, as well as blood capillaries.

On the other hand, bringing the sensor closer to the microneedle tip enables consistent in-vivo monitoring of biomarkers of clinical interest with reduced ISF volumes such as subnanoliter amounts ${ }^{8,16,29-31}$. The close proximity between the sensor and interstitium (containing ISF) ensures attaining an equilibrium protein concentration, as the biomarkers of clinical interest surrounding the sensor have a good correlation with the free ISF (and plasma) ${ }^{32}$. Such sensor configuration also enables reducing the lag time in ISF corresponding to changes of the blood glucose level associated with the capillary-to-sensor diffusion time $^{30}$. The device architecture mainly relies on an assembly process to place the biosensor compartment (i.e., electrodes of electrochemical transducers) inside the borehole of the hollow microneedle. By leveraging CMOS/MEMS technologies (e.g., through-silicon via (TSV) filling of copper or doped poly-silicon), metallization inside the hole for electrode fabrication can therefore be accomplished with wafer-level processes amenable to mass production ${ }^{33,34}$.

The state-of-the-art fabrication process for making silicon hollow microneedles typically relies on deepreactive ion etching (DRIE) of holes with small diameters from both sides because of the otherwise limited hole etching depth from a single side associated with so-called aspect ratio-dependent etching (ARDE) ${ }^{35-41}$. The challenge in this process is the need of precise double-sided alignment, especially with the ARDE-induced tapering hole profile ${ }^{41,42}$, and the significant hole widening during the needle sharpening process using isotropic wet etching. Alternatively, relatively large through-wafer holes are etched from one side through a combination of tapered and straight profiles ${ }^{43,44}$, whereas a smoothly tapered profile is more desirable ${ }^{45}$.

This paper presents the DRIE of silicon hollow microneedles, which resemble elongated cones with smooth tapering from the shank to extreme sharpness. A triplephase modified Bosch process was developed to enable the production of sufficiently deep holes with small diameters from a single side of the $\mathrm{Si}$ wafer. As such, more processing flexibility was provided for optimal pillar etching without the compromise of simultaneous pillar and hole etching. Afterward, the isotropic etching process was managed to selectively remove the unwanted silicon to open holes and to achieve needle sharpening. This process also prevents the holes from undesirable etching by the aggressive etchant.

\section{Design of THE microneedle chip}

Microneedle-enabled transdermal minimally invasive platforms typically utilize miniaturized needles of several hundred micrometers, resulting in a limited skin penetration depth to minimize the patient's discomfort. It is highly desirable to interrogate ISF in the superficial papillary dermis, which is directly beneath the epidermisdermis junction (with a variable depth of $100-200 \mu \mathrm{m}$ from the skin surface), without triggering the sensory nerve endings and blood capillaries in the deeper dermis (deeper papillary and reticular) ${ }^{24,46}$. Such pain-free ISF interrogation can be enabled by an array of $\sim 150 \mu \mathrm{m}$-long microneedles, which penetrate through the thinner epidermis (and stratum corneum) exactly, only touching the ISF-filled compartment. It is noteworthy that the optimization of the needle shape (e.g., via a smaller needle 


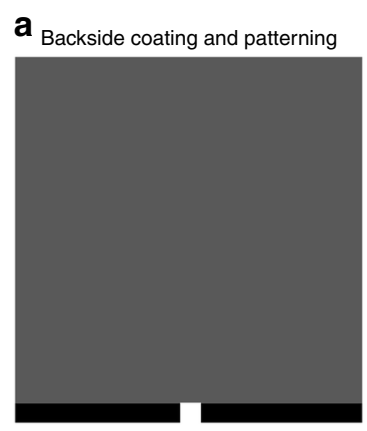

d



e Sharpening and hole opening



f

3D schematics
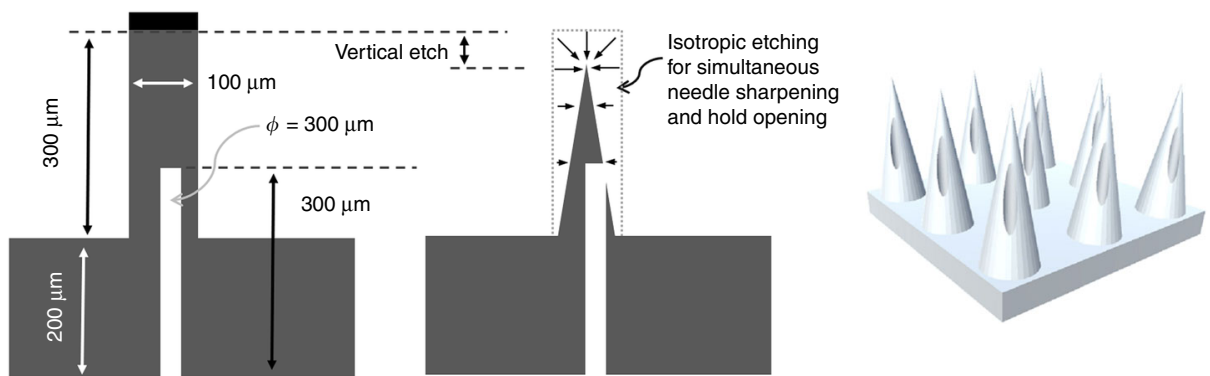

Fig. 1 Schematic fabrication process of silicon hollow microneedle arrays

base) can mitigate the incomplete penetration resulting from the so-called bed-nail effects and the viscoelastic nature of skin. Furthermore, fluid sampling approaches are prone to clogging associated with tissue coring at the insertion sites, which necessitates accurate placement of the borehole off-center from the needle axis ${ }^{44}$. This also enables improving the sharpness of the apex (essentially solid, rather than hollow) after the sharpening process.

Therefore, our microneedle array was designed to have silicon pillars of $200-300 \mu \mathrm{m}$ long to achieve the needle length of $\sim 150 \mu \mathrm{m}$ for etching vertically, along with lateral sharpening (see details in Fig. 1). Second, the diameter of the pillars was chosen as $100 \mu \mathrm{m}$ to provide sufficient mechanical strength for supporting skin penetration, as well as to enhance the penetration with a relatively small base in the combination of the smoothly tapered profile $^{7,44}$. Furthermore, the needle pitch of $300 \mu \mathrm{m}$ enables mitigation of the bed-nail effect, while maintaining the shear stress distribution during skin penetration to minimize needle breakage. For the hole-side etching, the $30 \mu \mathrm{m}$-diameter holes are designed to be anisotropically etched to $300 \mu \mathrm{m}$ deep (with an aspect ratio of 10) to provide sufficient overlap between the pillars and holes, which actually determines the opening location of the holes on the sidewalls of the sharpened needles. The borehole was intentionally shifted $30 \mu \mathrm{m}$ from the center of the column to mitigate tissue coring and improve the sharpness.
It is also noteworthy that a single or small number of needles frequently have clogging issues, wherein the microneedle bore is blocked by skin tissue. As such, an adequately large number of microneedles is necessitated to maximize the ISF access volume and consistency, given the requirements for a shallow penetration depth. The two-dimensional array design is also desirable to accomplish a multiplexed sensing system within a single chip for the simultaneous and selective screening of target biomarkers in ISF.

\section{Micromachining process}

Deep-reactive ion etching enables highly anisotropic silicon etching with high-selectivity relative to photoresists, making it feasible to fabricate structures with highaspect ratios (i.e., the ratio of depth to width) ${ }^{41}$. However, the etching rate rapidly decreases with increasing aspect ratios of the structures being etched, which is termed aspect ratio-dependent etching (ARDE) $)^{37,41,47}$. In this case, the etching rate rapidly decreases with the aspect ratio of the etched structures. Above a certain critical point of the aspect ratio, the etching rate reaches a constant extremely low value ${ }^{48}$. The proposed mechanism lies in that the ion flux to the bottom of the structure decreases along with its aspect ratio, resulting in insufficient passivation layer removal ${ }^{47}$. As such, holes with higher aspect ratios would start to pinch off at the bottom, and above a certain critical point of the aspect ratio, the 
Table 1 Parameters of the DRIE etching process with an RF frequency of $13.56 \mathrm{MHz}$

\begin{tabular}{|c|c|c|c|c|c|c|c|c|}
\hline & & ICP power (W) & Cycle (s) & Gas & $\begin{array}{l}\text { Flow Rate } \\
\text { (sccm) }\end{array}$ & RF power $(W)$ & $\begin{array}{l}\text { Pressure } \\
\text { (mTorr) }\end{array}$ & Etching rate \\
\hline \multirow[t]{2}{*}{ Standard Bosch at $15^{\circ} \mathrm{C}$} & Passivation & 1000 & 5 & $\mathrm{C}_{4} \mathrm{~F}_{8}$ & 160 & 5 & 20 & \multirow{2}{*}{$\begin{array}{l}1.8 \mu \mathrm{m} / \mathrm{min} \\
0.3 \mu \mathrm{m} / \mathrm{cycle}\end{array}$} \\
\hline & Etching & 1000 & 7 & $\mathrm{SF}_{6}$ & 160 & 20 & 25 & \\
\hline \multirow[t]{3}{*}{ Modified Bosch at $5^{\circ} \mathrm{C}$} & Passivation & 2500 & 0.6 & $\mathrm{C}_{4} \mathrm{~F}_{8}$ & 150 & Off & 60 & \multirow{2}{*}{$\begin{array}{l}7.8 \mu \mathrm{m} / \mathrm{min} \\
0.4 \mu \mathrm{m} / \mathrm{cycle}\end{array}$} \\
\hline & Depassivation & 2000 & 0.7 & $\begin{array}{l}\mathrm{SF}_{6} / \\
\mathrm{Ar}\end{array}$ & $200 / 30$ & 100 & 25 & \\
\hline & Etching & 2500 & 2 & $\mathrm{SF}_{6}$ & 400 & Off & 60 & \\
\hline
\end{tabular}

Note that the etching rate here was determined from the pillar structure etching with a large open area $(\sim 500 \mu \mathrm{m})$, and the etching rate can vary for different structures

etching rate would reach a constant extremely low value. The plasma provides energetic species (bombarding positive ions) that are accelerated toward the wafer surface by a strong electric field. To mitigate the effects of ARDE, an independent depassivation step was inserted into the standard dual-phase Bosch process (i.e., passivation and etching) $)^{49}$. In this step, the energetic ions (e.g., argon) directionally bombard the bottom of the etched holes, which are conformally deposited with a Teflon-type polymer during the preceding passivation step. Such a triple-phase DRIE (i.e., passivation, depassivation, etching) can efficiently remove the passivating polymers, enabling anisotropic etching with very high-aspect ratios and without undesirable tapered sidewall profiles.

In our experiment, the anisotropic etching of silicon structures (i.e., pillars, holes) was conducted in an inductively coupled plasma reactive ion etcher (ICP-RIE). Specifically, the pillars were etched with a standard dualphase Bosch DRIE recipe (i.e., passivation, etching) using an Oxford Instruments PlasmaLab 100 etcher. The holes were etched with a triple-phase modified Bosch recipe (i.e., passivation, depassivation, etching) using an Oxford Instruments PlasmaPro Estrelas100 etcher. The parameters of the "standard Bosch" recipe and the "modified Bosch" recipe are listed in Table 1 . The DRIE system is configured for 4-inch wafers, and smaller samples (e.g., square shapes with side lengths of $3-5 \mathrm{~cm}$ ) can be accommodated by mounting to a 4-inch silicon carrier wafer using Fomblin oil as a thermally conductive adhesive. The wafer is clamped with continuous helium backside cooling to ensure a constant wafer temperature, i.e., $15^{\circ} \mathrm{C}$ for the "standard Bosch" and $5{ }^{\circ} \mathrm{C}$ for the "modified Bosch" processing.

The microneedle pattern was fabricated using standard photolithography techniques on a (100) silicon wafer, with the major process steps schematically illustrated in Fig. 1. The Si wafers were cleaned by immersion into hydrofluoric acid (HF: deionized water $=1: 10$ ) for $30 \mathrm{~s}$, followed by deionized (DI) water rinsing, and nitrogen blow drying. First, a bilayer of AZ 4620 photoresist at a total thickness of $\sim 24 \mu \mathrm{m}$ was spun onto one side (termed as the 'backside") of a 4-inch wafer (double-side polished, Fig. 1a). Afterwards, ultraviolet (UV) light exposure was carried out with a mask aligner (Karl Suss MA6) at a dosage of $1600 \mathrm{~mJ} / \mathrm{cm}^{2}$, followed by immersing the exposed sample into a developer solution (AZ 400 K 4:1 diluted developer). The photolithography formed an array of holes in the photoresist of $\sim 30 \mu \mathrm{m}$ in diameter (Fig. 1a). Then, DRIE (i.e., the "modified Bosch" process) was performed to etch $300 \mu \mathrm{m}$-deep boreholes into the backside of the wafer, defining a high-aspect ratio (HAR) structure ( 1:10, Fig. 1b). Note that at this moment, the other side of the wafer (termed as 'frontside") was still flat. The DRIE process was halted before the boreholes were etched through the wafer to its frontside. Similar to the backside patterning, the frontside pillar pattern was defined (Fig. 1c) with alignment to the holes on the backside. The AZ 4620 photoresist was patterned to create cylindrical pillars aligned to the boreholes. This alignment also enabled accurate patterning of the holes such that their centers were offset from the needle axis. This offset was to address the tissue coring issue within the needle bore during insertion ${ }^{7}$. Pillars of $\sim 300 \mu \mathrm{m}$ in height and $100 \mu \mathrm{m}$ in diameter were then etched by DRIE (Fig. 1d), making an overlap of $100 \mu \mathrm{m}$ between the pillars and holes. At this point, the boreholes were still not exposed on the frontside. Essentially, they were still buried channels. Afterwards, using a mixed solution of hydrofluoric acid and nitric acid, the circular pillars were sharpened into conical needles, and the through-wafer holes were fully opened (Fig. 1e). This sharpening was realized by taking advantage of the isotropic etching nature of the chemical mixture, in which the etching rate decreases from the needle tip to the base ${ }^{50}$. Sharpening was also demonstrated by using the isotropic plasma dry etching and the combination of "wet" and "dry" isotropic etching. Holes 
were exposed on the sidewall of the needles, creating channels from the needles to the wafer's backside (Fig. 1f).

\section{Results and discussion Pillar etching}

The pillars were etched on a 4-inch Si wafer using dualphase "standard Bosch" processing with the parameters shown in Table 1 . The standard Bosch DRIE process was carried out for 300 cycles to etch $102 \mu \mathrm{m}$-high pillars with straight and smooth sidewall profiles (Fig. 2a). The pillar diameter was measured as $103 \mu \mathrm{m}$ with negligible pattern erosion from the designed $105 \mu \mathrm{m}$, showing high-fidelity pattern replication. Upon extending the etching for another 600 cycles, the pillar height increased to $297 \mu \mathrm{m}$, whereas the pillar top decreased to $87 \mu \mathrm{m}$, which was caused by resistance erosion (Fig. 2b). The pillar base decreased to $55 \mu \mathrm{m}$, creating a reentrant (negatively tapered) profile (i.e., the top is wider than the base) as a result of ARDE on large open areas ${ }^{51}$. It is believed to be very challenging to control the profile at heights $>100 \mu \mathrm{m}$ for features with large gaps. This is because the plasma sheath starts to follow the etched features and results in some nonvertical ions and, hence, increased undercutting.

Figure 3 compares the dependency of the etch depth and etch rate on the etch cycles in the etching of pillars and holes using dual-phase "standard Bosch" processing. The error bar represents the standard deviation from the average values. The etch depth in the pillar etching linearly increases with the etch cycles, showing an almost constant etch rate of $1.8 \mu \mathrm{m} / \mathrm{min}$. In contrast, the hole etching shows that the correlation between the etch depth and etch cycles gradually decreases (see supplementary Fig. 1), and hence, the etch rate decreases from $1.8 \mu \mathrm{m} /$ $\mathrm{min}$ to $1.1 \mu \mathrm{m} / \mathrm{min}$ in the etching of deeper holes $(>200 \mu \mathrm{m})$. The deceleration of the etching rate for highaspect ratio structures is attributed to the ARDE. The physical ion bombardment during the etching step is insufficient to remove the fluoropolymers on the bottom of the hole structures ${ }^{37}$. Moreover, a positively tapered profile (opposite to the reentrant sidewall) was also observed in the etching of the $400 \mu \mathrm{m}$-deep holes, resulting from severe mask erosion (even with a doublelayered photoresist) associated with the poor selectivity of the resist to $\mathrm{Si}$ (i.e., 1:18).

\section{Hole etching}

The dual-phase "standard Bosch" process consists of a passivation step and an etching step, suffering from ARDE for the etching of high-aspect ratio structures with wellcontrolled sidewall profiles. The triple-phase "modified Bosch" process has the distinctive feature of adding a depassivation step between the two steps of the standard Bosch. The depassivation step utilizes energetic Ar ions to efficiently remove the fluorocarbon passivating polymers (see details in Table 1). As such, the DRIE process provided the highly directional (almost completely anisotropic) etching of holes of $200 \mu \mathrm{m}$ in depth and $30 \mu \mathrm{m}$ in diameter. The etching mask on the wafer surface is faithfully replicated in the underlying silicon (Fig. 4a). The thicknesses of the photoresist (single layer) before and after the DRIE were respectively $10.65 \mu \mathrm{m}$ and $9.10 \mu \mathrm{m}$. The selectivity of the photoresist with respect to $\mathrm{Si}$ was



Fig. 3 The dependency of the etch depth and etch rate on the etch cycles using "standard Bosch" DRIE processing for the fabrication of pillars and holes. The error bar represents the standard deviation from the average values


Fig. 2 Pillar etching with a 300 cycles and b 900 cycles using the "standard Bosch" DRIE processing 

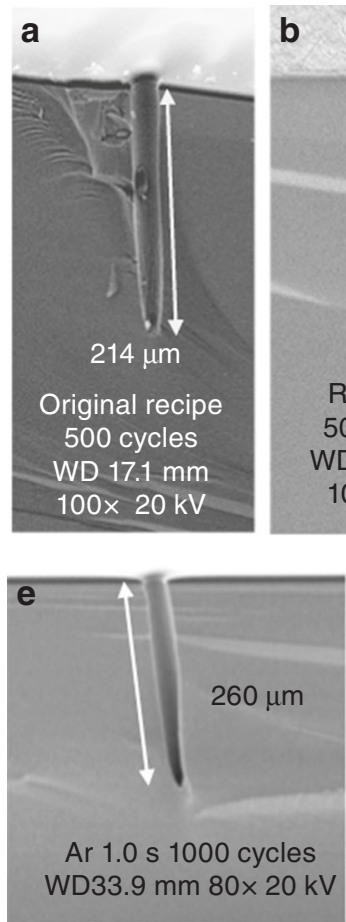

b
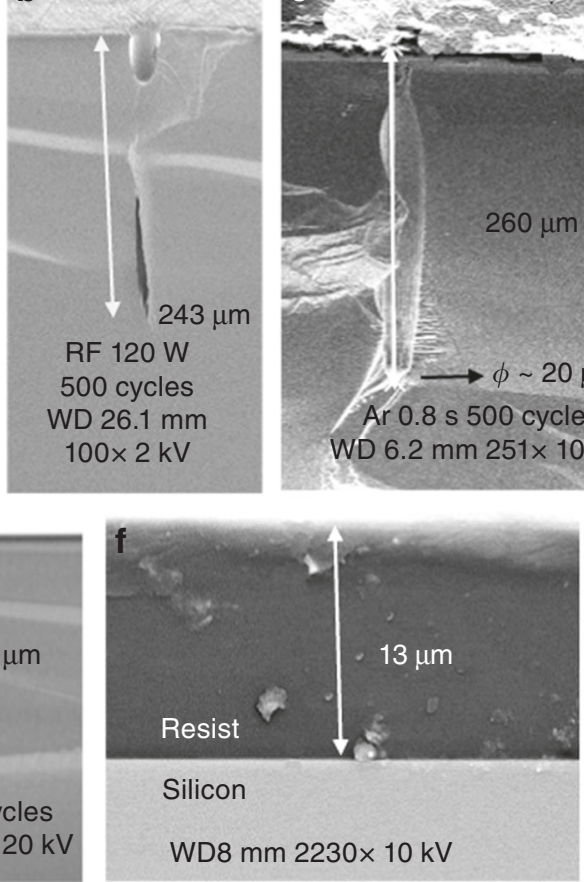
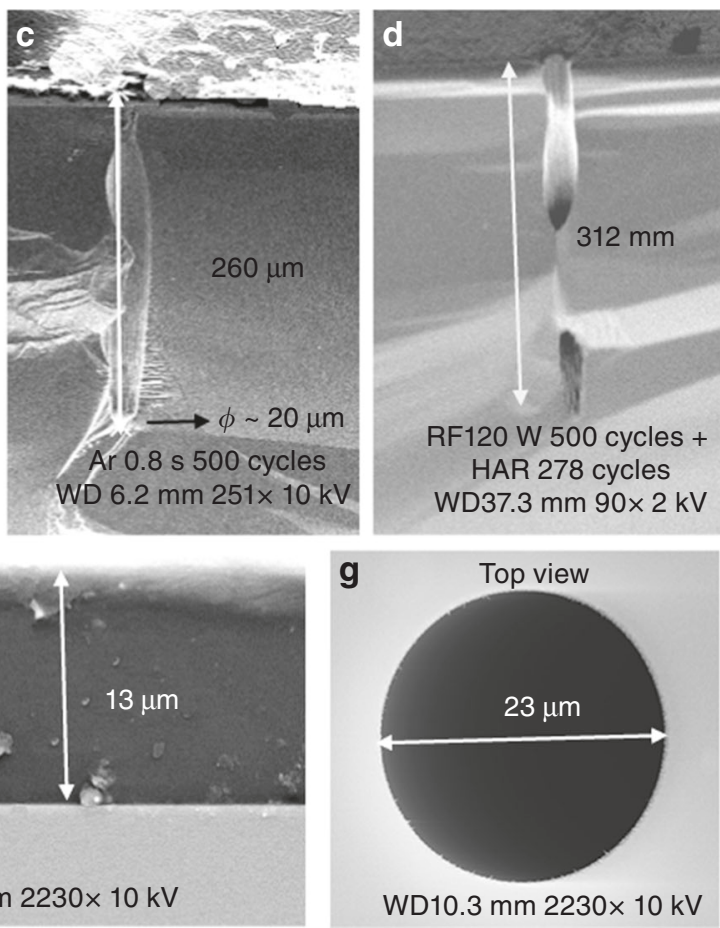

Fig. 4 The optimization of hole DRIE etching using triple-phase "modified Bosch" processing

thus $1: 200$, with a Si etching rate of $12 \mu \mathrm{m} / \mathrm{min}$. Note that this etching also has the advantage of smaller scalloping due to the shorter etch cycle, resulting in a smoother surface that is beneficial to potential processing for the integration of sensing elements.

The etching of deeper holes $(>200 \mu \mathrm{m})$ was also observed to decelerate as a result of insufficient passivation layer removal, similar to the case for etching with the dual-phase "standard Bosch" process. However, the triplephase "modified Bosch" process enables adjusting the parameters of the independent depassivation (Ar ion bombardment) step for mitigating ARDE. To promote ion bombardment, the RF power (and hence, the acceleration voltage of the argon ions, Fig. $4 \mathrm{~b}$ ) and the Ar ion bombardment time (Fig. 4c) were respectively increased to enhance the removal of the passivating polymers at the bottoms of the holes (illustrated in Fig. 1b). As such, the etch depth increased without degrading the desired feature, especially upon using the combination of the original recipe and the HAR recipe (Fig. $4 \mathrm{~d}$ ). In another experiment, etching with the depassivation step of $1 \mathrm{~s}$ was carried out for 1000 cycles (Fig. 4e). The remaining photoresist was still sufficiently thick under the extended long ion bombardment (Fig. 4f), showing negligible pattern erosion (Fig. 4g). Note that the etching depth with a specific recipe has a standard deviation of $<5 \mu \mathrm{m}$, showing high reproducibility.

\section{Needle sharpening}

The silicon micropillars were then sharpened into conical needles using an isotropic etching process (Fig. 5). This wet chemical etching of silicon utilizes a so-called HNA system, consisting of hydrofluoric acid (HF), nitric acid $\left(\mathrm{HNO}_{3}\right)$, and a comparatively weak acetic acid $\left(\mathrm{CH}_{3} \mathrm{COOH}\right)$, which can be replaced with water ${ }^{50,52}$. The overall reaction involves the oxidation of silicon to $\mathrm{SiO}_{2}$ by $\mathrm{HNO}_{3}$ and subsequent $\mathrm{SiO}_{2}$ dissolution in HF. It is important that the overall reaction is limited by the diffusion of HF, and as a result, a ratio of 19:1 (which was adopted in this work) between $\mathrm{HNO}_{3}$ and $\mathrm{HF}$ was chosen to assure that the oxide formation dominates its removal. By placing the silicon micropillar sample at the bottom of a static solution, the HF diffusion to the silicon surface was made significantly slower than the dissolution reaction at the surface, and hence, it was the rate-limiting factor. HF reacts with $\mathrm{SiO}_{2}$ when they are in contact, rapidly consuming $\mathrm{HF}$ in the process. Owing to the large amount of exposed $\mathrm{Si}$ (and $\mathrm{SiO}_{2}$ ) between the pillars, the reactive species are significantly consumed at the pillar bottom, rather than at the pillar top. As the reaction proceeds in a static solution, the bottom has less replenishment than does the top, especially when the solution depth is well-controlled, which will result in faster etching (shrinking) of the pillar top. Another factor also contributes to the faster etch rate at the top-the fact that the 



Fig. 5 The solid microneedle sharpening process using isotropic wet etching in a mixed solution of nitric acid and hydrofluoric acid

edge is essentially exposed to HF from both vertical and lateral directions, whereas such etching will stop until a rounded (rather than sharp) shape is achieved.

In the isotropic wet etching experiment, square samples $(1-2 \mathrm{~cm}$ side length) were used, and 600 cycles of "standard Bosch" DRIE etching was performed to fabricate solid micropillars. A 5 min wet etching resulted in a nearly vertical pillar of $94 \mu \mathrm{m}$ wide and $217 \mu \mathrm{m}$ high (Fig. 5a). Upon extending the etching time to $20 \mathrm{~min}$, the pillar shrunk to $58 \mu \mathrm{m}$ in diameter at the top and $200 \mu \mathrm{m}$ in height (Fig. 5b), with the etching rate of appx. $1 \mu \mathrm{m} / \mathrm{min}$ in good agreement with the literature ${ }^{50,52}$. Upon adding another $20 \mathrm{~min}$ of etching, the blunt tip was sharpened into a single point of $<1 \mu \mathrm{m}$, and simultaneously, the pillar height shrunk to $152 \mu \mathrm{m}$ (Fig. 5c). By keeping the solution under static conditions (i.e., without agitation), as the etching proceeds, the etching species only diffuse from the bulk solution surrounding the silicon sample to replenish the solution contained within the spaces between the pillars. As a result, the sharpening shows high uniformity over the entire sample, with a percentage standard deviation of $<5 \%$ for needle length (Fig. 5d), except in rows $1-3$ on the sample edge, where the lateral diffusion from the adjacent open space becomes as important as the vertical diffusion (Fig. 5d). To achieve a higher degree of spatial uniformity across an entire wafer (including the edge), it is suggested to incorporate sacrificial structures (e.g., borders, extra rows of lines on the edge) to adjust the local concentration of etchant ${ }^{50}$.

\section{Simultaneous needle sharpening and hole opening}

Afterwards, the micropillars incorporating holes were sharpened using a mixed solution of $\mathrm{HNO}_{3}$ and $\mathrm{HF}$ under stationary conditions. The isotropic etching-in particular, the etching from the lateral direction-simultaneously sharpened the needle tip and exposed the buried channel enclosed within the pillar. In such a manner, $160 \mu \mathrm{m}$-high hollow microneedles were fabricated after 40 min wet etching, with a percentage standard deviation of $2.7 \%$, expressing high uniformity (Fig. 6a). Additionally, $30 \mu \mathrm{m}$-diameter holes were positioned off-center to mitigate the tissue coring issue during needle penetration, leading to so-called snake-fang needles ${ }^{7}$ (Fig. 6b). A similar shape was also fabricated by using the combination of isotropic "wet" and plasma "dry" etchings, with the needle height showing a percentage standard deviation of $1.5 \%$ (Fig. 6c). Here, the micropillars were etched in the HNA solution for $30 \mathrm{~min}$ to obtain a blunt tip (inset Fig. 6c), followed by a $15-\mathrm{min} \mathrm{SF}_{6}$ plasma etching in a reactive-ion etching system (Phantom II, Trion Technology Inc.). A $\mathrm{SF}_{6}$ plasma etching process alone for $35 \mathrm{~min}$ (without wet etching) with the PlasmaLab 100 etcher, in which the $\mathrm{C}_{4} \mathrm{~F}_{8}$ gas was deactivated from the "standard Bosch" recipe, was also demonstrated to be capable of needle sharpening, with the needle height showing a percentage standard deviation of $1.3 \%$ (Fig. 6d). The aggressive plasma etching resulted in a nearly straight sidewall with a smoothly tapered profile and a narrow base to mitigate the incomplete needle penetration 



Fig. 6 Hollow microneedle sharpening process using various methods. The hollow microneedle sharpening process using wet etching (a, b), a combination of wet etching and plasma etching (c), and plasma etching only (d). The inset in Fig. $6 \mathrm{c}$ shows the microneedle after wet etching and before plasma etching (scale bar $100 \mu \mathrm{m}$ )

associated with skin elasticity ${ }^{27,28}$. Some of the major advantages of using plasma etching for needle sharpening include better process control and easy automation, as well as the elimination of handling strong oxidants.

\section{Capillary filling}

The capillary filling of DRIE-etched through-silicon holes ( $40 \mu \mathrm{m}$-diameter, without needle features) has been demonstrated using 1\% weight/volume Allura red dye solution $(0.1 \mathrm{~g}$ dye $+10 \mathrm{~mL}$ water, Fig. 7a). Oxygen plasma treatment is imperative to assure a hydrophilic surface with a water contact angle of $<20$ degrees $^{53}$ by removing the passivating polymer (i.e., $\mathrm{C}_{4} \mathrm{~F}_{8}$ ) left over from the previous Bosch DRIE process. The authors also observed that the lateral spreading of a water droplet was significantly larger on the $\mathrm{Si}$ surface after the oxygen plasma treatment. The sample with through-wafer holes was placed on the polished side of a clean silicon wafer (treated with the $\mathrm{O}_{2}$-plasma, Fig. 7a), creating a micrometer-level gap between the two surfaces to ensure effective capillary uptake. By placing a drop of the red dye solution in proximity to the hole chip (as indicated by the dashed circle in Fig. 7a), water uptake by the DRIE-etched through-wafer holes was accomplished in $\sim 1-2 \mathrm{~s}$, changing the color from white (empty holes, Fig. $7 \mathrm{~b}$ inset) to red (Fig. 7b). The top surface with the polymer coating (without $\mathrm{O}_{2}$ plasma treatment) is hydrophobic, so the droplet on the top cannot spread out laterally. Upon placing a nitrocellulose paper on the top of the hole chip with a gentle finger push, the nitrocellulose paper was able to wick liquid through its capillary structure (Fig. 7a). This result shows the feasibility of direct integration between $\mathrm{Si}$ microneedles and paper microfluidics for the purpose of lateral-flow assay assembly.

\section{Skin penetration}

Afterwards, the skin penetrability was assessed with excised porcine skin $^{54}$. Porcine skin is often used as a model of human skin due to the similarities in anatomy such as the thickness of the stratum corneum. The insertion force is suggested to range from 0.1 to $3 \mathrm{~N}$, such that a thumb-push is sufficient for skin penetration, rather than requiring aid from impact-insertion applicators ${ }^{7,28}$. A razor was used to remove the subcutaneous fat on the back of the skin, making 3-4 mm-thick porcine skin samples. Then, the skin with good surface conditions (without hair or skin disorders such as scarring) was cut into square pieces $(2-3 \mathrm{~cm}$ length) for experimentation. Following IPA soaking, the microneedle chip was gently thumb-pushed onto the porcine skin sample for a few 

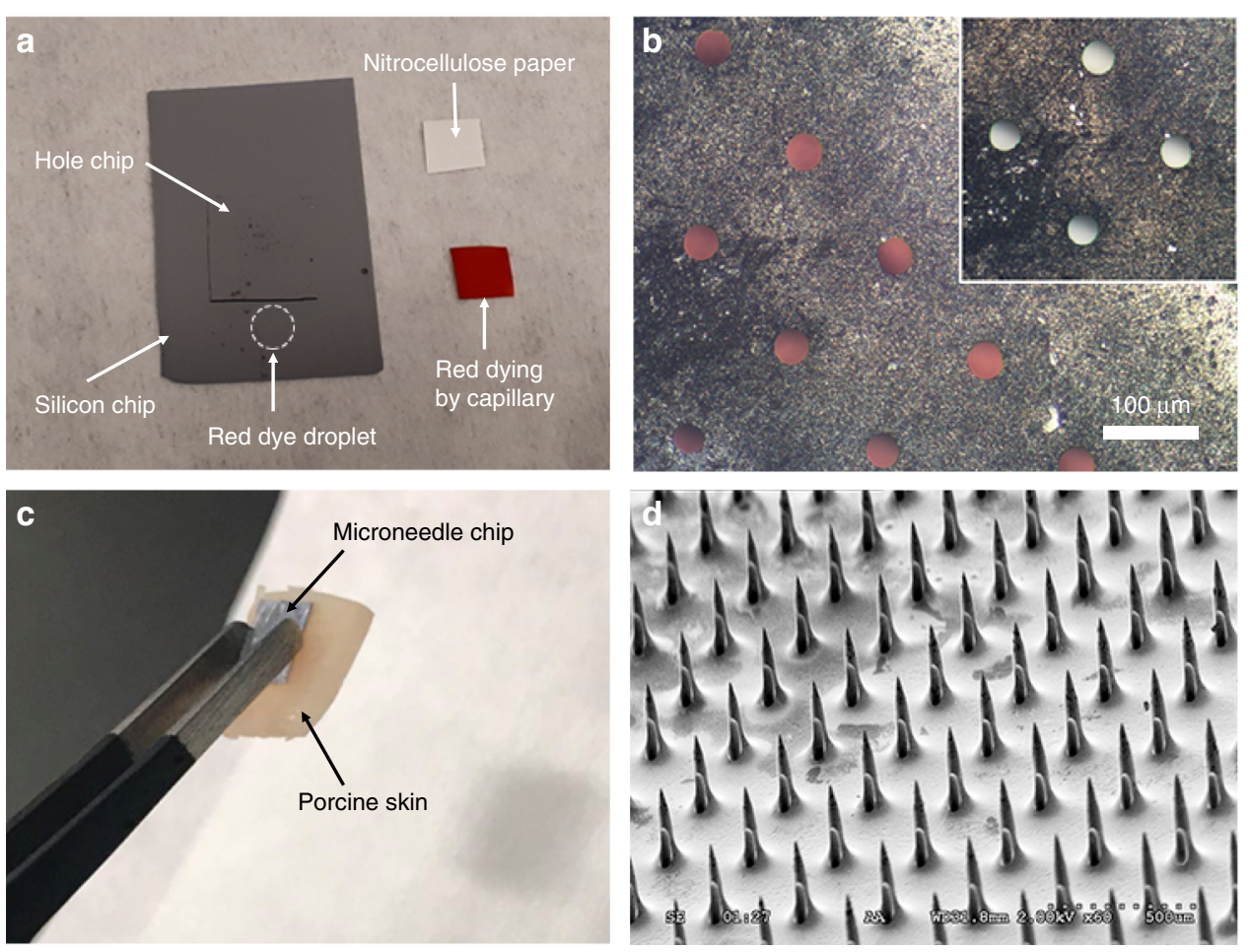

Fig. 7 Capillary filling of DRIE-etched through-silicon holes and microneedle penetration into porcine skin. a Capillary filling of DRIE-etched through-silicon holes with a water solution of red dye and the microscopic images of holes $\mathbf{b}$ with capillary filling (inset: empty holes before filling). $\mathbf{c}$ Microneedle penetration into porcine skin; $\mathbf{d}$ microneedles remaining mechanically intact after repetitive penetrations

seconds, and the needles were capable of holding the skin sample (Fig. 7c), indicating successful skin penetration. The successful skin penetration is largely attributed to the extreme sharpness of the microneedles, as the insertion force linearly decreases with the interfacial area of the needle tip, according to a study ${ }^{28}$. Following separation from the skin, the microneedle chip was baked at $250{ }^{\circ} \mathrm{C}$ for $30 \mathrm{~min}$ on a hotplate for disinfection and sterilization by a burning (oxidation) process, while keeping the residual body tissues adsorbed onto the needle shank during skin penetration. This step also dried the sample to avoid contaminating the SEM chamber. Afterward, we carried out an scanning electron microscopy (SEM) inspection, in which we did not observe any mechanical failures. As such, the microneedles were concluded to be mechanically intact after repetitive skin penetration (Fig. 7d). This agrees well with the theoretical prediction regarding similar Si structures, in which the Si pillar fracture force was an order of magnitude greater than the insertion force necessary for skin penetration ${ }^{28,44}$. Note that the blackcolored substances on the needle shanks are likely the burning products of residual body tissues.

\section{Conclusion}

A DRIE process for fabricating silicon hollow microneedle arrays has been presented, aiming to explore the feasibility of microneedle-enabled in-vivo biosensors, in which the sensing elements can be incorporated on the inner surface the boreholes. The insertion of a depassivation process using directional Ar ion bombardment into the two standard Bosch half-cycles resulted in efficient removal of the passivating fluorocarbon layer, enabling highly anisotropic etching of circular holes with diameters as small as $30 \mu \mathrm{m}$ to a depth of $>300 \mu \mathrm{m}$. The needle tips were sharpened to single points with radii as small as $5 \mu \mathrm{m}$ using either wet or dry plasma etching or the combination of both. This isotropic etching step also opened the holes originally embedded within the pillars. Such sharp microneedles have been demonstrated to be sufficiently robust to penetrate porcine skin without needing a mechanical applicator, with the needles remaining mechanically intact after repetitive penetrations. Capillary filling of the DRIE-etched through-silicon holes has also been demonstrated, showing their feasibility for transporting biomarkers of clinical interest to the sensing sites using capillary action.

\section{Acknowledgements}

The work was funded by ExVivo Labs Inc., OCE and NSERC. The University of Waterloo's Quantum NanoFab cleanroom was used for the microneedle fabrication. This infrastructure would not be possible without the significant contributions from CFREF, CFI, Industry Canada, the Ontario Ministry of 
Research \& Innovation and Mike \& Ophelia Lazaridis. Their support is gratefully acknowledged.

\section{Conflict of interest}

The authors declare that they have no conflict of interest.

Supplementary information accompanies this paper at https://doi.org/ 10.1038/s41378-019-0077-y.

Received: 29 December 2018 Revised: 10 April 2019 Accepted: 12 April 2019

Published online: 26 August 2019

\section{References}

1. Kim, D. et al. Epidermal electronics. Sci. (80-). 333, 838-843 (2011).

2. Gao, W. et al. Fully integrated wearable sensor arrays for multiplexed in situ perspiration analysis. Nature 529, 509-514 (2016).

3. Lee, $H$. et al. A graphene-based electrochemical device with thermoresponsive microneedles for diabetes monitoring and therapy. Nat. Nanotechnol. 11, 566-572 (2016).

4. Barry, B. W. Transdermal drug delivery. Nat. Biotechnol. 26, 565-597 (2007).

5. Samant, P. P. \& Prausnitz, M. R. Mechanisms of sampling interstitial fluid from skin using a microneedle patch. Proc. Natl Acad. Sci. 115, 4583-4588 (2018).

6. Kolluru, C., Williams, M., Chae, J. \& Prausnitz, M. R. Recruitment and collection of dermal interstitial fluid using a microneedle patch. Adv. Healthc. Mater. 8 1801262 (2019).

7. Mukerjee, E. V., Collins, S. D., Isseroff, R. R. \& Smith, R. L. Microneedle array for transdermal biological fluid extraction and in situ analysis. Sens. Actuators, A Phys. 114, 267-275 (2004).

8. Windmiller, J. R. et al. Bicomponent microneedle array biosensor for minimallyinvasive glutamate monitoring. Electroanalysis 23, 2302-2309 (2011).

9. Tilli, M. et al. Handbook of Silicon Based MEMS Materials and Technologies (Elsevier, Oxford, UK, 2015).

10. Kotzar, G. et al. Evaluation of MEMS materials of construction for implantable medical devices. Biomaterials 23, 2737-2750 (2002).

11. Kang, S. et al. Bioresorbable silicon electronic sensors for the brain. Nature $\mathbf{5 3 0}$ 71-76 (2016)

12. Hwang, S. et al. Dissolution chemistry and biocompatibility of single-crystalline silicon nanomembranes and associated materials for transient electronics. ACS Nano 8, 5843-5851 (2014).

13. Norman, J. J. et al. Reliability and accuracy of intradermal injection by Mantoux technique, hypodermic needle adapter, and hollow microneedle in pigs. Drug Deliv. Transl. Res. 4, 126-130 (2014).

14. Hochberg, L. R. et al. Neuronal ensemble control of prosthetic devices by a human with tetraplegia. Nature 442, 164-171 (2006).

15. Wang, P. C. et al. Fabrication and characterization of polymer hollow microneedle array using UV lithography into micromolds. J. Micro. Syst. 22, 1041-1053 (2013).

16. Ranamukhaarachchi, S. A., Padeste, C., Dübner, M. \& Häfeli, U. O. Integrated hollow microneedle-optofluidic biosensor for therapeutic drug monitoring in sub-nanoliter volumes. Sci. Rep. 6, 29075 (2016).

17. Campbell, P. K., Jones, K. E., Huber, R. J., Horch, K. W. \& Normann, R. A. A siliconbased, three-dimensional neural interface: manufacturing processes for an intracortical electrode array. IEEE Trans. Biomed. Eng. 38, 758-768 (1991).

18. Wise, Q. B. K. D. Single-unit neural recording with active microelectrode arrays. IEEE Trans. Biomed. Eng. 48, 911 (2001).

19. Song, S. et al. A capacitive biosensor for cancer diagnosis using a functionalized microneedle and a 13.7b-resolution capacitance-to-digital converter from 1 to 100nF. in 2019 IEEE International Solid- State Circuits Conference(ISSCC) 194-196 (IEEE, San Francisco, US, 2019).

20. Shenoy, $\mathrm{V}$. et al. A CMOS analog correlator-based painless nonenzymatic glucose sensor readout circuit. IEEE Sens. J. 14, 1591-1599 (2014).

21. Niedzwiecki, M. M. et al. Human suction blister fluid composition determined using high- resolution metabolomics. Anal. Chem. 90, 3786-3792 (2018).

22. Tran, B. Q. et al. Proteomic characterization of dermal interstitial fluid extracted using a novel microneedle-assisted technique. J. Proteome Res. 17, 479-485 (2018).
23. Jina, A. et al. Design, development, and evaluation of a novel microneedle array-based continuous glucose monitor. J. Diabetes Sci. Technol. 8, 483-487 (2014).

24. Ventrelli, L., Strambini, L. M. \& Barillaro, G. Microneedles for transdermal biosensing: current picture and future direction. Adv. Healthc. Mater. 4, 2606-2640 (2015).

25. Wiig, H. \& Swartz, M. A. Interstitial fluid and lymph formation and transport: physiological regulation and roles in inflammation and cancer. Physiol. Rev. 92, 1005-1060 (2012).

26. Benias, P. C. et al. Structure and distribution of an unrecognized interstitium in human tissues. Sci. Rep. 8, 4947 (2018).

27. Crichton, M. L., Chen, X., Huang, H. \& Kendall, M. A. F. Elastic modulus and viscoelastic properties of full thickness skin characterised at micro scales. Biomaterials 34, 2087-2097 (2013).

28. Davis, S. P., Landis, B. J., Adams, Z. H., Allen, M. G. \& Prausnitz, M. R. Insertion of microneedles into skin: measurement and prediction of insertion force and needle fracture force. J. Biomech. 37, 1155-1163 (2004).

29. Ribet, F., Stemme, G. \& Roxhed, N. Ultra-miniaturization of a planar amperometric sensor targeting continuous intradermal glucose monitoring. Biosens. Bioelectron. 90, 577-583 (2017).

30. Ribet, F., Stemme, G. \& Roxhed, N. Real-time intradermal continuous glucose monitoring using a minimally invasive microneedle-based system. Biomed. Micro. 20, 101 (2018).

31. Iniewski, K. VLSI Circuits for Biomedical Applications (Artech House, Norwood, MA, USA, 2008).

32. Sibley, L. \& Aukland, K. Wick sampfing of interstitial fluid in rat skin: further analysis and modifications of the method. Microvasc. Res. 49, 39-49 (1986).

33. Koester, S. J. et al. Wafer-level 3D integration technology. IBM J. Res. Dev. 52, 583-597 (2008)

34. Vereecken, P. M., Binstead, R. A., Deligianni, H. \& Andricacos, P. C. The chemistry of additives in damascene copper plating. IBM J. Res. Dev. 49, 3-18 (2005).

35. Taylor, H. K., Sun, H., Hill, T. F., Farahanchi, A. \& Boning, D. S. Characterizing and predicting spatial nonuniformity in the deep reactive ion etching of silicon. $J$. Electrochem. Soc. 153, C575 (2006).

36. Wu, B., Kumar, A. \& Pamarthy, S. High aspect ratio silicon etch: a review. J. Appl. Phys. 108, 051101 (2010).

37. Lai, S. L., Johnson, D. \& Westerman, R. Aspect ratio dependent etching lag reduction in deep silicon etch processes. J. Vac. Sci. Technol. A Vac., Surf., Film. 24, 1283-1288 (2006)

38. Gardeniers, H. J. G. E. et al. Silicon micromachined hollow microneedles for transdermal liquid transport. J. Micro. Syst. 12, 855-862 (2003).

39. Stoeber, B. \& Liepmann, D. Arrays of hollow out-of-plane microneedles for drug delivery. J. Micro. Syst. 14, 472-479 (2005).

40. Griss, P. \& Stemme, G. Side-opened out-of-plane microneedles for microfluidic transdermal liquid transfer. J. Micro. Syst. 12, 296-301 (2003).

41. Tang, Y., Sandoughsaz, A., Owen, K. J. \& Najafi, K. Ultra deep reactive ion etching of high aspect-ratio and thick silicon using a ramped-parameter process. J. Micro. Syst. 27, 686-697 (2018).

42. Shul, R. J. \& Pearton, S. J. Handbook of Advanced Plasma Processing Techniques (Springer-Verlag, Berlin Heidelberg, 2000).

43. Roxhed, N., Samel, B., Nordquist, L., Griss, P. \& Stemme, G. Painless drug delivery through microneedle-based transdermal patches featuring active infusion. IEEE Trans. Biomed. Eng. 55, 1063-1071 (2008).

44. Roxhed, N., Gasser, T. C., Griss, P., Holzapfel, G. A. \& Stemme, G. Penetrationenhanced ultrasharp microneedles and prediction on skin interaction for efficient transdermal drug delivery. J. Micro. Syst. 16, 1429-1440 (2007).

45. Chua, B., Desai, S. P., Tierney, M. J., Tamada, J. A. \& Jina, A. N. Physical Effect of microneedles shape on skin penetration and minimally invasive continuous glucose monitoring in vivo. Sens. Actuators A. Phys. 203, 373-381 (2013).

46. Johansson, O., Wang, L. X., Hilliges, M. \& Liang, Y. Intraepidermal nerves in human skin: PGP 9.5 immunohistochemistry with special reference to the nerve density in skin from different body regions. J. Peripher. Nerv. Syst. 4, 43-52 (1999)

47. Abdolvand, R. \& Ayazi, F. An advanced reactive ion etching process for very high aspect-ratio sub-micron wide trenches in silicon. Sens. Actuators, A Phys. 144, 109-116 (2008).

48. Yeom, J., Wu, Y., Selby, J. C. \& Shannon, M. A. Maximum achievable aspect ratio in deep reactive ion etching of silicon due to aspect ratio dependent transport and the microloading effect. J. Vac. Sci. Technol. B Microelectron. Nanom. Struct. 23, 2319 (2005). 
49. Blauw, M. A., Craciun, G., Sloof, W. G., French, P. J. \& van der Drift, E. Advanced time-multiplexed plasma etching of high aspect ratio silicon structures. J. Vac. Sci. Technol. B Microelectron. Nanom. Struct. 20, 3106 (2002).

50. Bhandari, R., Negi, S., Rieth, L. \& Solzbacher, F. A wafer-scale etching technique for high aspect ratio implantable MEMS structures. Sens. Actuators, A Phys. 162 130-136 (2010).

51. Walker, M. J. Comparison of Bosch and cryogenic processes for patterning high-aspect-ratio features in silicon. in Proc. SPIE 4407, MEMS Design,
Fabrication, Characterization, and Packaging. 89-99 (2001). https://doi.org/ $10.1117 / 12.425288$

52. Schwartz, B. \& Robbins, H. Chemical etching of silicon. J. Electrochem. Soc. Solid State Sci. Technol. 123, 1903-1909 (1976).

53. Kaya, S. et al. A systematic study of plasma activation of silicon surfaces for self assembly. ACS Appl. Mater. Interfaces 7, 25024-25031 (2015).

54. Wang, P. C., Paik, S. J., Kim, S. H. \& Allen, M. G. Hypodermic-needle-like hollow polymer microneedle array: fabrication and characterization. J. Micro. Syst. 23 991-998 (2014). 